\title{
Quintessence from the decay of superheavy dark matter
}

\author{
Houri Ziaeepour* \\ Mullard Space Science Laboratory, Holmbury St. Mary, Dorking, Surrey RH5 6NT, United Kingdom
}

(Received 29 August 2003; published 18 March 2004)

\begin{abstract}
We investigate the possibility of replacing the cosmological constant with a gradual condensation of a scalar field produced during the decay of a superheavy dark matter. The advantage of this class of models over ordinary quintessence is that the evolution of the dark energy and the dark energy are correlated and the cosmological coincidence problem is solved. This model does not need a special form for the quintessence potential; even a simple $\phi^{4}$ theory or an axionlike scalar is enough to explain the existence of dark energy. We show that the model has intrinsic feedback between the energy density of dark matter and the scalar field such that for a large volume of the parameter space, the equation of state of the scalar field from very early in the history of the Universe is very close to a cosmological constant. Other aspects of this model are consistent with recent cosmic microwave background and large scale structure observations.
\end{abstract}

DOI: 10.1103/PhysRevD.69.063512

PACS number(s): 98.80.Cq, 95.35.+d

\section{INTRODUCTION}

Quintessence models are alternatives to a cosmological constant, i.e., a nonzero vacuum energy density. They are not, however, flawless. Even in models with tracking solutions, the potential of the scalar field must somehow be finetuned to explain its smallness and its slow variation until today. In addition, many of them cannot address the coincidence problem, i.e., why the density of dark matter (DM) and dark energy (DE) evolve in such a way that they become comparable just after galaxy formation.

Recently, a number of authors have proposed an interaction between dark matter and the quintessence field to explain the coincidence. Chimento et al. [1], based on an earlier work by Chimento et al. [2] and Zimdahl et al. [3], suggest an asymptotic scaling law between the density of DE and DM. In their model due to a dissipative interaction between dark matter and the quintessence scalar field $\phi_{q}$, $\rho_{\mathrm{DM}} / \rho_{q} \rightarrow$ cte, where $\rho_{\mathrm{DM}}$ and $\rho_{q}$ are, respectively, DM and the scalar field density. Assuming this "strong coincidence" [1], they find the class of potentials $V_{q}\left(\phi_{q}\right)$ such that the equation of state has a solution with scaling behavior. Then, using constraints from nucleosynthesis, they find that this category of models has $w_{q} \geq-0.7$. This value is marginally compatible with the Wilkinson microwave anisotropy probe (WMAP) data and far from publicly available supernova type Ia (SN-Ia) data which prefer $w_{q} \sim-1$. In another version of the same model, Zimdahl et al. [3] consider a nonstatic scaling solution, $\rho_{\mathrm{DM}} / \rho_{q} \propto\left(a_{0} / a\right)^{\eta}$. The model with $\eta=1$ solves the coincidence paradigm, but the standard cold dark matter model with a cosmological constant $(\Lambda \mathrm{CDM})$ fits the SN-Ia data better and their best fit has $w_{q} \sim-0.7$.

Amendola et al. [4] have extensively studied the interaction of quintessence field and dark matter in models with tracking solutions and $w_{q}>-1$. They show that these models are equivalent to a Brans-Dicke Lagrangian with powerlaw potential and look like a "fifth force." Modification of the CMB anisotropy spectra by such interactions is observ-

*Email address: hz@mssl.ucl.ac.uk able and puts stringent constraints on their parameters.

Comelli et al. [5] study a model in which the effect of interaction between quintessence scalar and dark matter appears as time dependence of DM particle mass. This explains the extreme adjustment of dark matter and dark energy densities during cosmological evolution. The coupling between two fields increases the parameter space for both and reduces by orders of magnitude the amount of fine-tuning. In this respect, as we will see below, their model is similar to what we propose in this work. However, there are a number of issues that these authors have not addressed. Cosmological observations put strict limits on the variation of fundamental parameters including the DM mass. In their model, the largest amount of variation happens around and after the matterdomination epoch. The mass variation must leave an imprint on the $\mathrm{CMB}$ and large-structure formation which was not observed.

In addition to the lack of explanation for the coincidence in many quintessence models, it is difficult to find a scalar field with necessary characteristics in the frame of known particle physics models without some fine-tuning of the potential [6]. In general, it is assumed that the quintessence field is an axion with high-order, thus nonrenormalizable, interactions with the standard model particles (or its supersymmetric extension), which is highly suppressed at low energies. However, Chung et al. [7] show that any supergravity-induced interaction between $\phi_{q}$ and other scalars with a vacuum expectation value (VEV) of the order of the Planck mass can increase the very tiny mass of the $\phi_{q}\left(m_{q} \sim H_{0} \sim 10^{-33} \mathrm{eV}\right)$ expected in many models, unless a discrete global symmetry prevents their contribution to the mass.

In a very recent work, Farrar and Peebles [8] study models with a Yukawa interaction between DM and the quintessence scalar field. Like the Comelli et al. model, this interaction affects the mass of the dark matter particles. The general behavior of these models is close to $\Lambda \mathrm{CDM}$ with some differences which can distinguish them. One of the special cases with a two-component CDM imitates the $\Lambda$ CDM very closely. Many aspects of this model is similar to the model studied in the present work, but without consider- 
ing the source of the intimate relation between DM and DE in contrast (we believe) to the present work. Moreover, the necessity of having a very special self-interaction potential for the quintessence field is not removed.

What we propose here is a model for dark energy somehow different from previous quintessence models (a preliminary investigation of this model has been presented in [9]). We assume that DE is the result of the condensation of a scalar field produced during the very slow decay of a massive particle. In most of the quintessence models, the scalar field is largely produced during inflation or a reheating period, such that to control its contribution to the total energy of the Universe, its potential must be a negative exponential (in most cases the sum of two exponentials) or a negative power function [6]. We show that in the present model, a very small production rate of the scalar field replaces the fine-tuning of the potential, and practically any scalar field even without a self-interaction has a tracking solution for a large part of its parameter space.

The main motivation for this class of models is the possibility of a top-down solution [10-12] for the mystery of ultrahigh energy cosmic rays (UHECR's) [13-15]. If a very small part of the decay remnants which make the primaries of UHECR's is composed of a scalar field $\phi_{q}$, its condensation can have all the characteristics of a quintessence field. We show that in this model the most natural equation of state for the quintessence scalar is very close to a cosmological constant, at least until the age of the Universe is much smaller than the lifetime of the superheavy dark matter (SDM, Wimpzilla) which is the origin of the quintessence field.

Another motivation is the fact that a dark energy with $w_{q} \lesssim-1$ fits the SN-Ia data better than a cosmological constant [16-18]. Although the sensitivity of CMB data to the equation of state of the dark energy is much less than SN's, with 95\% confidence WMAP data give the range -1 \pm 0.22 for the $w_{q}[19,20]$. Estimation from galaxy clusters evolution is also in agreement with this range [21]. On the other hand, it has been demonstrated that the cosmological equation of state for decaying dark matter in the presence of a cosmological constant is similar to quintessence with $w_{q}$ $\leqslant-1$ [18]. Both observations therefore seem to encourage a top-down solution which explains simultaneously the dark energy and the UHECR's.

Like other models with interaction between DM and DE, the coincidence in this model is solved without fine-tuning. Parameters can be changed by many orders of magnitude without destroying the general behavior of the equation of state or the extreme relation between the energy density of dark energy and the total energy density in the early Universe.

In Sec. II we solve the evolution equations for dark matter and dark energy. For two asymptotic regimes we find analytical solutions for the evolution of $\phi_{q}$. In Sec. III we present the results of the numerical solution of the evolution equations including baryonic matter, and we show that both approaches lead essentially to the same conclusion. We study also the extent of the parameter space. The effect of DM anisotropy on the energy density of the dark energy is stud- ied in Sec. IV. We show that the perturbation of dark energy in this model is very small and very far from the resolution of present or near-future observations. The late-time decoherence of the scalar field is discussed in Sec. II A. We give a qualitative estimation of the necessary conditions and leave a proper investigation of this issue as well as the possible candidates for $\phi_{q}$ to future works.

\section{COSMOLOGICAL EVOLUTION OF DECAYING DARK MATTER AND A QUINTESSENCE FIELD}

Consider that at a very early epoch in the history of the Universe, just after inflation, the cosmological "soup" consists of two species: a superheavy dark matter (SDM) $-X$ particles-decoupled from the rest of the "soup" since very early time, and a second component, which we do not consider in detail. The only constraint we require is that it must consist of light species including baryons, neutrinos, photons, and light dark matter (by light we mean with respect to $X)$. For simplicity, we assume that $X$ is a scalar field $\phi_{x}$. Considering $\phi_{x}$ to be a spinor or vector does not change the general conclusions of this work. We also assume that $\phi_{x}$ is quasistable, i.e., its lifetime is much longer than the present age of the Universe. A very small part of its decay remnants is considered to be a scalar field $\phi_{q}$ with negligibly weak interaction with other fields.

The effective Lagrangian can be written as

$$
\begin{aligned}
\mathcal{L}= & \int d^{4} x \sqrt{-g}\left[\frac{1}{2} g^{\mu \nu} \partial_{\mu} \phi_{x} \partial_{\nu} \phi_{x}\right. \\
& \left.+\frac{1}{2} g^{\mu \nu} \partial_{\mu} \phi_{q} \partial_{\nu} \phi_{q}-V\left(\phi_{x}, \phi_{q}, J\right)\right]+\mathcal{L}_{J} .
\end{aligned}
$$

The field $J$ presents collectively other fields. The term $V\left(\phi_{x}, \phi_{q}, J\right)$ includes all interactions including the selfinteraction potential for $\phi_{x}$ and $\phi_{q}$,

$$
V\left(\phi_{x}, \phi_{q}, J\right)=V_{q}\left(\phi_{q}\right)+V_{x}\left(\phi_{x}\right)+g \phi_{x}^{m} \phi_{q}^{n}+W\left(\phi_{x}, \phi_{q}, J\right) .
$$

The term $g \phi_{x}^{m} \phi_{q}^{n}$ is important because it is responsible for the annihilation of $X$ and back reaction of the quintessence field by reproducing them. $W\left(\phi_{x}, \phi_{q}, J\right)$ presents interactions which contribute to the decay of $X$ to light fields and to $\phi_{q}$ [in addition to what is shown explicitly in Eq. (2)]. The very long lifetime of $X$ constraints this term and $g$. They must be strongly suppressed. For $n=2$ and $m=2$, the $g$ term contributes to the mass of $\phi_{x}$ and $\phi_{q}$. Because of the huge mass of $\phi_{x}$ (which must come from another coupling) and its very small occupation number $\left\langle\phi_{x}^{2}\right\rangle \sim 2 \rho_{x} / m_{x}^{2}$, for sufficiently small $g$ the effect of this term on the mass of the SDM is very small. We discuss the role of this term in detail later. If the interaction of other fields with $\phi_{q}$ is only through the exchange of $X$ (for instance due to a conserved symmetry shared by both $X$ and $\phi_{q}$ ), the huge mass of $X$ suppresses the interaction and therefore the modification of their mass. This solves the problem of "fifth force" in the dark [4] and the SM sectors. 
In a homogeneous universe the evolution equations for $\phi_{q}$ and $\phi_{x}$ are

$$
\begin{aligned}
& \ddot{\phi}_{q}+3 H \dot{\phi}_{q}+\frac{\partial V}{\partial \phi_{q}}=0, \\
& \ddot{\phi}_{x}+3 H \dot{\phi}_{x}+\frac{\partial V}{\partial \phi_{x}}=0,
\end{aligned}
$$

where a dot denotes the comoving time derivative. In the rest of this work, we treat $\phi_{x}$ and $J$ as classical particles and deal only with their density and equation of state. We assume that $X$ particles are nonrelativistic (i.e., part of the CDM) with negligible self-interaction, i.e.,

$$
V_{x}\left(\phi_{x}\right)=\frac{1}{2} m_{x}^{2} \phi_{x}^{2}
$$

Under this assumption, $\phi_{x}$ can be replaced by

$$
\phi_{x} \sim\left(\frac{2 \rho_{x}}{m_{x}^{2}}\right)^{1 / 2}
$$

If $X$ is a spinor, the lowest-order (Yukawa) interaction term in Eq. (1) is $g \phi_{q} \bar{\psi} \psi$. In the classical treatment of $X$,

$$
\bar{\psi} \psi \sim \frac{\rho_{x}}{m_{x}} .
$$

The same argument about the negligible effect of the interaction on the mass of DM and SM particles is applied. For simplicity, we consider only the scalar case.

For potential $V_{q}\left(\phi_{q}\right)$ we consider a simple $\phi^{4}$ model,

$$
V_{q}\left(\phi_{q}\right)=\frac{1}{2} m_{q}^{2} \phi_{q}^{2}+\frac{\lambda}{4} \phi_{q}^{4}
$$

Conservation of energy-momentum, Einstein, and dynamic equations gives the following system of equations for the fields:

$$
\begin{aligned}
\dot{\phi}_{q}\left[\ddot{\phi}_{q}+3 H \dot{\phi}_{q}+m_{q}^{2} \phi_{q}+\lambda \phi_{q}^{3}\right]=-2 g \dot{\phi}_{q} \phi_{q}\left(\frac{2 \rho_{x}}{m_{x}^{2}}\right)+\Gamma_{q} \rho_{x} \\
\dot{\rho}_{x}+3 H \rho_{x}=-\left(\Gamma_{q}+\Gamma_{J}\right) \rho_{x}-\pi^{4} g^{2}\left(\frac{\rho_{x}^{2}}{m_{x}^{3}}-\frac{\rho_{q}^{\prime 2}}{m_{q}^{3}}\right) \\
\dot{\rho}_{J}+3 H\left(\rho_{J}+P_{J}\right)=\Gamma_{J} \rho_{x}, \\
H^{2}=\left(\frac{\dot{a}}{a}\right)^{2}=\frac{8 \pi G}{3}\left(\rho_{x}+\rho_{J}+\rho_{q}\right) \\
\rho_{q}=\frac{1}{2} m_{q}^{2} \dot{\phi}_{q}^{2}+\frac{1}{2} m_{q}^{2} \phi_{q}^{2}+\frac{\lambda}{4} \phi_{q}^{4}
\end{aligned}
$$

where Eq. (10) is the Boltzmann equation for $X$ particles. We calculate its right-hand side in the Appendix. $\rho_{q}^{\prime}$ is the density of quintessence particles (not the classical field $\phi_{q}$ ) with an average energy larger than $m_{x}$ in the local inertial frame. Only interaction between these particles contributes to the reproduction of SDM. $\Gamma_{q}$ and $\Gamma_{J}$ are, respectively, the decay width of $X$ to $\phi_{q}$ and to other species. In Eq. (9) we have replaced $\phi_{x}$ with its classical approximation from Eq. (6). The effect of the decay Lagrangian $W\left(\phi_{x}, \phi_{q}, J\right)$ appears as $\left(\Gamma_{q}+\Gamma_{J}\right) \rho_{x}$, which is the decay rate of $X$ particles [see Eq. (A9) in the Appendix].

At very high temperatures when $\rho_{x} \gg \pi^{4} g^{2} m_{x}^{3} \Gamma$, the annihilation and reproduction terms in Eq. (10) are dominant. $X$ particles, however, are nonrelativistic up to temperatures close to their rest mass. Quintessence scalar particles at this time are relativistic and therefore their density falls faster than SDM density by a factor of $a(t)$. The probability of annihilation also decreases very rapidly. Consequently, from a very early time only the decay term in Eq. (10) is important. The dominance of annihilation/reproduction can happen only if the production temperature of $X$ particles, i.e., preheating/reheating temperature, is very high. Such scenarios, however, can make a dangerous amount of gravitinos [22]. For this reason, presumably the reheating temperature must be much smaller than $m_{x}$ and annihilation dominance never happens. This cannot put the production of SDM in danger because it has been shown [23] that even with a very low reheating temperature, they can be produced. It seems, therefore, reasonable to study the evolution of the fields only when the annihilation/reproduction is negligible. Another reason for this simplification is that we are interested in the decohered modes of $\phi_{q}$. When the self-annihilation of $X$ particles is the dominant source of $\phi_{q}$, most particles are highly relativistic and their self-interaction does not have time to make long-wavelength modes. This claim needs, however, a detailed investigation of the process of decoherence, which we leave for another work.

The system of equations (9)-(13) is highly nonlinear and an analytical solution cannot be found easily. There are, however, two asymptotic regimes which permit an approximate analytical treatment. The first one happens at very early time just after the production of $X$ (presumably after preheating $[11,12])$ and the decoherence of $\phi_{q}$ 's long-wavelength modes. In this epoch, $\phi_{q} \sim 0$ and can be neglected. The other regime is when comoving time variation of $\phi_{q}$ is very slow and one can neglect $\ddot{\phi}_{q}$. We show that the first regime leads to a saturation (tracking) solution where $\phi_{q} \rightarrow$ cte. It can then be treated as the initial condition for the second regime when $\phi_{q}$ changes slowly.

The effect of the last term on the right-hand side of Eq. (10) as we argued is negligible. The solution of Eq. (10) is then straightforward,

$$
\rho_{x}(t)=\rho_{x}\left(t_{0}\right) e^{-\Gamma\left(t-t_{0}\right)}\left(\frac{a\left(t_{0}\right)}{a(t)}\right)^{3},
$$

where $\Gamma \equiv \Gamma_{q}+\Gamma_{J}$ is the total decay width of $X$. We consider $t_{0}$ to be the time after production and decoupling of $X$. These two times can be very different, but with an extremely long 
lifetime for $X$ and its weak interaction with other species, it is not important which one of them is selected as $t_{0}$.

After inserting the solution (14) and neglecting all the terms proportional to $\phi_{q}$, Eq. (9) simplifies to

$$
\dot{\phi}_{q} \frac{d}{d t}\left(a^{3} \dot{\phi}_{q}\right)=\Gamma_{q} a^{3}\left(t_{0}\right) \rho_{x}\left(t_{0}\right) e^{-\Gamma\left(t-t_{0}\right)}
$$

and can be solved

$$
\begin{aligned}
\frac{1}{2} \dot{\phi}_{q}^{2}(t) & \equiv K_{q}(t) \\
& =\left(\frac{a\left(t_{0}\right)}{a(t)}\right)^{6}\left[K_{q}\left(t_{0}\right)+\Gamma_{q} \rho_{x}\left(t_{0}\right) \int_{t_{0}}^{t} d t \frac{a^{3}}{a\left(t_{0}\right)} e^{-\Gamma\left(t-t_{0}\right)}\right] .
\end{aligned}
$$

For $a \propto t^{k}$, the integral term in Eq. (16) decreases with time (i.e., $\ddot{\phi}_{q}<0$ ). This means that after a relatively short time $\phi_{q}$ is saturated and its density does not change, in other words it behaves like a cosmological constant. The numerical simulation in the next section confirms this result. If $\phi_{q}$ was a classical field, the natural choice for the initial value of the kinetic energy $K_{q}\left(t_{0}\right)$ was $K_{q}\left(t_{0}\right)=0$ assuming a very rapid production of $X$. However, in reality $\phi_{q}$ is a quantum field and it gets time to decohere and to settle as a classical field. The initial value of $K_{q}\left(t_{0}\right)$ can therefore be nonzero. Its exact value can only be determined by investigating the process of decoherence. In any case with the expansion of the Universe, its effect on $\dot{\phi}_{q}$ decreases very rapidly because of the $a^{-6}(t)$ factor in Eq. (16).

Next we consider the regime where $\phi_{q}$ changes very slowly and we can neglect $\ddot{\phi}_{q}$ and higher orders of $\dot{\phi}_{q}$. Equation (9) gets the following simplified form:

$$
\dot{\phi}_{q}\left(m_{q}^{2} \phi_{q}+\lambda \phi_{q}^{3}\right)=-2 g \dot{\phi}_{q} \phi_{q}\left(\frac{2 \rho_{x}}{m_{x}^{2}}\right)+\Gamma_{q} \rho_{x} .
$$

We expect that self-interaction of $\phi_{q}$ is much stronger than its coupling to $X$. Neglecting the first term in the right-hand side of Eq. (17), its $\phi_{q}$-dependent part can be integrated,

$$
\frac{d}{d t}\left(\frac{1}{2} m_{q}^{2} \phi_{q}^{2}+\frac{\lambda}{4} \phi_{q}^{4}\right)=\frac{d V}{d t}\left(\phi_{q}\right)=\Gamma_{q} \rho_{x},
$$

which is then easily solved,

$$
V_{q}\left(\phi_{q}\right)=V_{q}\left[\phi_{q}\left(t_{0}^{\prime}\right)\right]+\Gamma_{q} \rho_{x}\left(t_{0}^{\prime}\right) \int_{t_{0}^{\prime}}^{t} d t\left(\frac{a\left(t_{0}^{\prime}\right)}{a(t)}\right)^{3} e^{-\Gamma\left(t-t_{0}^{\prime}\right)} .
$$

Here $V_{q}$ is the potential energy of $\phi_{q}$. From Eqs. (18) and (19) it is clear that the final value of the potential and therefore $\phi_{q}$ energy density is driven by the decay term and not the self-interaction. Therefore, the only vital condition for this model is the existence of a long life SDM and not the potential of $\phi_{q}$.
In Eq. (19) the initial values $t_{0}^{\prime}$ and $\phi_{q}\left(t_{0}^{\prime}\right)$ are different from Eq. (16). They correspond to the time and to the value of $\phi_{q}$ in the first regime when it approaches saturation. Similar to Eq. (16), the time dependence of $\phi_{q}$ in Eq. (19) vanishes exponentially and the behavior of $\phi_{q}$ approaches a cosmological constant.

To estimate the asymptotic value of $\phi_{q}$, we assume that $a(t) \propto t^{k}$. Using Eq. (19) with the additional assumption that $t_{s}-t_{0}^{\prime} \ll 1 / \Gamma\left(t_{s}\right.$ is the saturation time $)$, we find

$$
V\left(\phi_{q}\right)-V\left[\phi_{q}\left(t_{0}^{\prime}\right)\right] \sim \frac{\Gamma_{q} \rho_{x}\left(t_{0}^{\prime}\right)}{(3 k-1)}\left[1-\left(\frac{t_{0}^{\prime}}{t}\right)^{(3 k-1)}\right] .
$$

If we define the saturation time as the time when $V\left(\phi_{q}\right)$ $-V\left[\phi_{q}\left(t_{0}^{\prime}\right)\right]$ has $90 \%$ of its final value, for $t_{s} \ll t_{\text {eq }}$ with $t_{\text {eq }}$ the matter-radiation equilibrium time, $k=\frac{1}{2}$ and

$$
t_{s} \sim 100 t_{0}^{\prime} .
$$

For $t_{s} \gg t_{\mathrm{eq}}, k=\frac{2}{3}$ and

$$
t_{s} \sim 10 t_{0}^{\prime} .
$$

The interesting conclusion one can make from Eq. (20) is that the initial density of SDM, its production time, and its decay rate to $\phi_{q}$, which are apparently independent quantities, determine together the final value of the dark energy density. The long lifetime of SDM is expected to be due to a symmetry which is broken only by nonrenormalizable highorder weak-coupling operators. They become important only at very large energy scales. These conditions are exactly what is needed to have a small dark energy density according to Eq. (20). In Sec. III we see that numerical calculation confirms these results.

We can also observe here the main difference between this model and other quintessence models. If $\phi_{q}$ is produced during, e.g., the decay of inflation or from the decay of a short-lived particle in the early Universe, its final density should be much larger than observed dark energy unless either its production width was fine-tuned to unnaturally small values or its self-interaction was exponentially suppressed with some fine-tuning of its rate.

\section{A. Decoherence}

Decoherence of scalar fields has been mainly studied in the context of phase transition [24] in a thermal system. Examples are phase transition in condensed matter $[24,25]$ and before, during, and after inflation in the early Universe $[26,27]$. In the latter case, the aim is to study the inflation itself, production of defects, and reheating. Decoherence is the result of self-interaction as well as interaction between a field (regarded as the order parameter after decoherence) and other fields in the environment. Long-wavelength modes behave like a classical field, i.e., they do not show "particlelike" behavior if quantum correlation between modes is negligible. More technically this happens when the density matrix for these modes is approximately diagonal. It has been shown [26] that interaction with higher modes is enough to decohere long-wavelength modes (see Calzetta et al. [27] for a review). The classical order parameter corresponds to these modes after their decoherence. One can consider a cutoff in the mode space which separates the sys- 

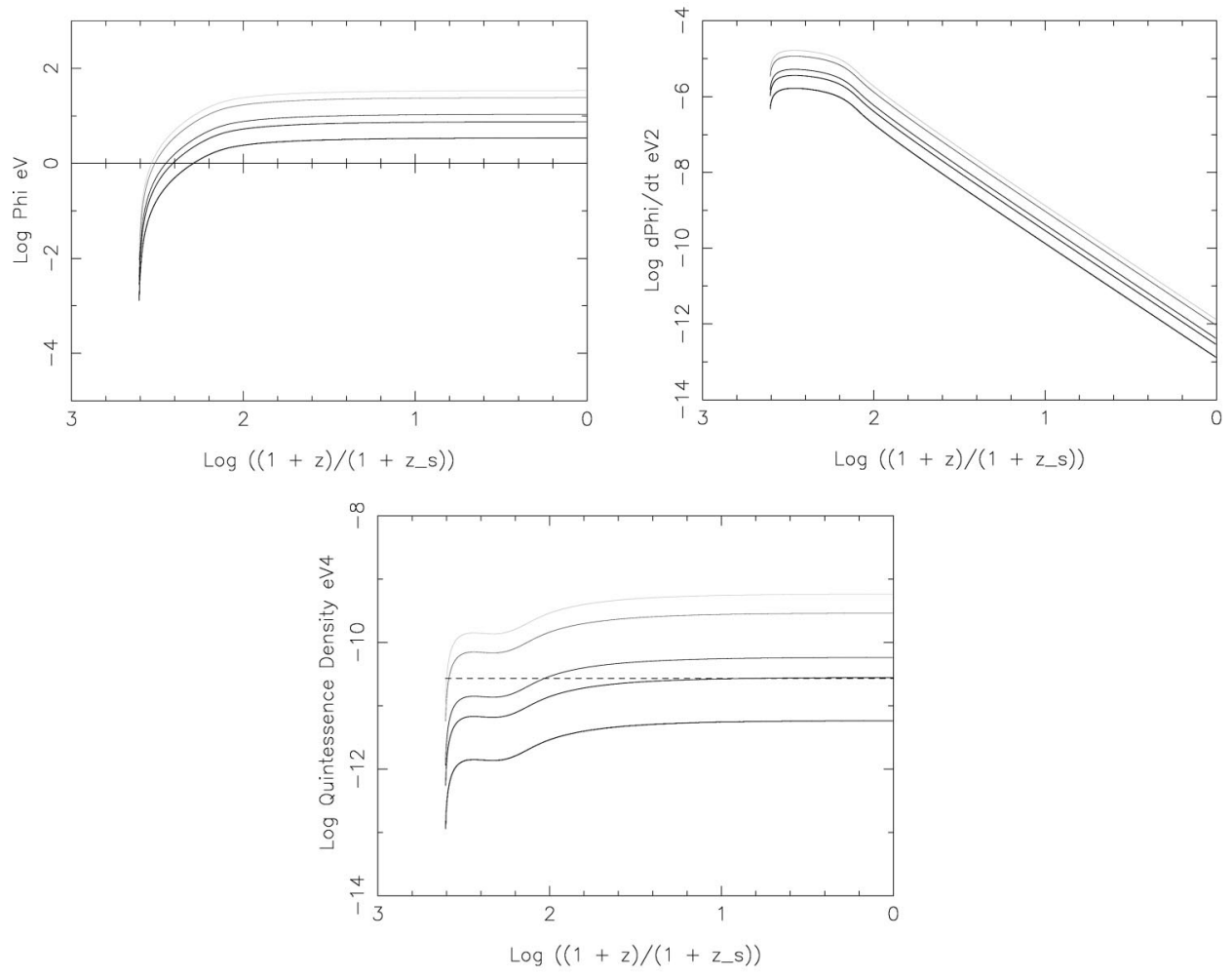

FIG. 1. Evolution of quintessence field (top left), its derivative (top right), and its total energy density (bottom): from bottom to top (dark to light gray), $\Gamma_{0}$ $\equiv \Gamma_{q} / \Gamma=10^{-16}, \quad 5 \Gamma_{0}, \quad 10 \Gamma_{0}$, $50 \Gamma_{0}, 100 \Gamma_{0}$. The dashed line is the observed value of the dark energy. $m_{q}=10^{-6} \mathrm{eV}, \lambda=10^{-20}$. tem (i.e., long-wavelength modes) from the environment (short wavelengths). The cutoff can be considered as an evolving scale which determines at each cosmological epoch the decoherent/coherent modes [27].

It has been shown [25] that the decoherence time in a thermal phase transition is shorter than the spinodal time, i.e., the time after the beginning of the phase transition when the scalar field or more precisely $\left\langle\phi^{2}\right\rangle$ settles at the minimum of the potential. The decoherence time in the presence of external fields (with couplings of the same order as selfinteraction) is

$$
t_{d} \sim \frac{1}{m} .
$$

By replacing Minkovski time with conformal time and considering a time-dependent cutoff $[26,27,25]$ one can show that modes with

$$
\frac{k^{2}}{a^{2}}+m^{2} \lesssim H^{2}
$$

decohere and behave like a classical scalar field. The effect of the coupling constant is logarithmic and less important.

If the SDM exists, it is produced during preheating [12] just after the end of the inflation presumably at $T$ $\sim 10^{14}-10^{16} \mathrm{eV}$, which corresponds to

$$
H \sim 10^{-6}-10^{-4} \mathrm{eV} .
$$

From Eq. (21) this time range permits scalars with mass $m$ $\lesssim 10^{-6} \mathrm{eV}$ to decohere. When the size of the Universe get larger, $\phi_{q}$ stops decohering. This also helps having a very small dark energy density. If the preheating/reheating had happened when the Hubble constant was smaller, them $m_{q}$ also must be smaller to have long-wavelength modes which can decohere. We will see in the next section that in this case the main term in the $V\left(\phi_{q}\right)$ potential is the self-interaction. Moreover, $\lambda$ can be larger, which helps a faster decoherence of long-wavelength modes.

The argument given here is evidently very qualitative and needs much deeper investigation. In the present work, we take the possibility of decoherence as granted and study the evolution of $\phi_{q}$ as a classical scalar field.

\section{NUMERICAL SOLUTION}

To have a better understanding of the behavior and the parameter space of this model, we have solved Eqs. (9)-(13) numerically. We have also added the interaction between various species of the standard model particles to the simulation to be closer to real cosmological evolution and to obtain the equation of state of the remnants. This is especially important for constraining the lifetime of SDM [28]. Without considering the interaction between high-energy remnants and the rest of the SM particles, especially the CMB, the lifetime of SDM must be orders of magnitude larger than the present age of the Universe.

Details of interaction simulation are discussed in [28] and we do not repeat them here. The Boltzmann equation for SM species [Eq. (1) in [28]] replaces Eq. (11). Because of numerical limitations we switch on interactions only from $z$ $=10^{9}$ downward. For the same reason, we had to begin the simulation of $X$ decay from $z \sim 10^{14}$, which is equivalent to a temperature of $T=10^{11} \mathrm{eV}$. The expected reheating temperature is model-dependent and varies from $\sim 10^{22} \mathrm{eV}$ to 

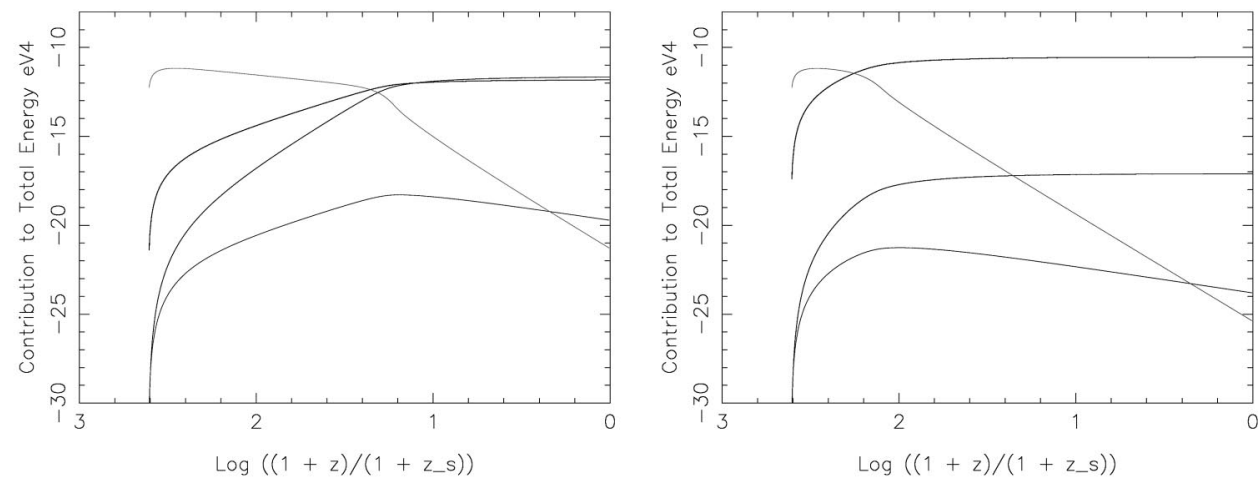

FIG. 2. Evolution of the contribution to the total energy density of $\phi_{q}$ for $\Gamma_{0} \equiv \Gamma_{q} / \Gamma=10^{-16}$. Top left: $m_{q}=10^{-8} \mathrm{eV}$ and $\lambda$ $=10^{-20}$; top right: $m_{q}=10^{-6} \mathrm{eV}$ and $\lambda=10^{-20}$; bottom: $m_{q}$ $=10^{-6} \mathrm{eV}$ and $\lambda=10^{-10}$. From dark to light gray curves are: mass, self-interaction, interaction with SDM, and kinetic energy.

$\sim 10^{7} \mathrm{eV}$. For the time being no observational constraint on this large range is available. The change in the initial temperature, however, does not modify the results of the simulation significantly if $f_{q} \equiv \Gamma_{q} / \Gamma$ is rescaled inversely proportional to redshift and to the total decay width $\Gamma$, and proportional to $m_{x}$. In other words, two models lead to very similar results for the quintessence field if

$$
\frac{f_{q}}{f_{q}^{\prime}}=\frac{z^{\prime} \Gamma^{\prime} m_{x}}{z \Gamma m_{x}^{\prime}} .
$$

For the lifetime of $X$ we use the results from [28] and [18], which show that a lifetime $\tau=5 \tau_{0}-50 \tau_{0}\left(\tau_{0}\right.$ the present age of the Universe) can explain the observed flux of UHECR's as well as the cosmic equation of state with $w_{q} \lesssim-1$. In the following, we consider $\tau=5 \tau_{0}$. Our test shows that increasing $\tau$ to $50 \tau_{0}$ does not significantly modify the extent of the admissible parameter space or other main characteristics of the dark energy model. We consider only the models with $m=2, n=2$, and $g=10^{-15}$ in Eq. (2). The results for $10^{-20}$ $\leqslant g \leqslant 10^{-5}$ are roughly the same as what we present in this section, and therefore they are not shown. The discussion in Sec. II as well as Fig. 2 show that the contribution of the interaction with the SDM in the total energy density of $\phi_{q}$ is much smaller than other terms.

Figure 1 shows the evolution of $\phi_{q}$, its time derivative, and its total energy density from the end of $X$ production to saturation redshift $z_{s}$. Here we have used as $z_{s}$ the redshift after which up to simulation precision the total energy density of $\phi_{q}$ does not change anymore. The result of the simulation is quite consistent with the approximate solutions discussed in Sec. II. The final density energy of $\phi_{q}$ is practically proportional to $\Gamma_{q} / \Gamma$. The latter quantity encompasses three important parameters of the model: The fraction of energy of the remnants, which changes to $\phi_{q}$; the fraction of energy in the long-wavelength modes, which can decohere; and the coupling of these modes to the environment, which contributes to $\phi_{q}$ yield and to the effective formation redshift of the classical quintessence field $\phi_{q}$. Therefore, the effective volume of the parameter space presented by this simulation is much larger and the fine-tuning of parameters is much less than what is expected from just one parameter.

Figure 2 shows the evolution in the contribution of different terms of the Lagrangian (1) to the total energy of $\phi_{q}$. Very soon after beginning production of the quintessence field, the potential takes over the kinetic energy and the latter begins to decrease. The relative contribution of each term and their time of dominance, as this figure demonstrates, depend on the parameters, especially $m_{q}$ and $\lambda$. Another conclusion from this plot is that changing these parameters by orders of magnitude does not change the general behavior of the model significantly, and for a large part of the parameter space the final density of quintessence energy is close to the observed value. This can also be seen in Fig. 3 and Fig. 4, where the evolution of quintessence energy is shown for various combinations of parameters.

\section{PERTURBATIONS}

Large- and medium-scale observations show that the dark energy is quite smooth and uncorrelated from the clumpy dark matter [29]. If the DE origin is the decay of the dark matter, the question arises whether it clumps around dark matter halos or has a large-scale perturbation which is not observed in the present data. In this section, we investigate the evolution of spatial perturbations in $\phi_{q}$ and show that 

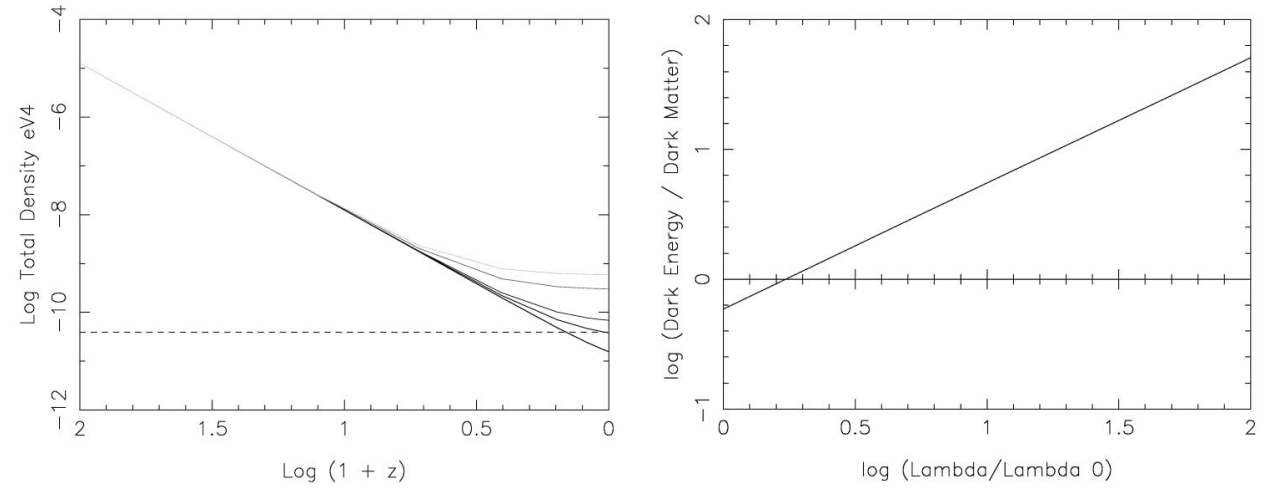

FIG. 3. Left: evolution of the total density with redshift: from bottom to top (dark to light gray), $\Gamma_{0} \equiv \Gamma_{q} / \Gamma=10^{-16}, 5 \Gamma_{0}, 10 \Gamma_{0}$, $50 \Gamma_{0}, 100 \Gamma_{0}$. The dashed line is the observed value of the dark energy. $m_{q}=10^{-6} \mathrm{eV}, \quad \lambda=10^{-20}$. Right: relative density of dark energy and CDM as a function of $\Gamma_{q} / \Gamma$. The x-axis is normalized to $\Gamma_{0} \equiv \Gamma_{q} / \Gamma=10^{-16}$.

they decrease with time. Another area of interest in doing such an exercise is to investigate any imprint of the model on the power spectrum of matter and the CMB anisotropy.

We use the synchronous gauge metric,

$$
d s^{2}=d t^{2}-a^{2}(t)\left(\delta_{i j}-h_{i j}\right) d x^{i} d x^{j} .
$$

For small spatial fluctuations $\phi_{q}(x, t)=\bar{\phi}_{q}(t)+\delta \phi_{q}(x, t)$, where from now on barred quantities are the homogeneous component of the field depending only on $t$. We define the same decomposition for other fields.

We consider only scalar metric fluctuations $h \equiv \delta^{i j} h_{i j}$ and neglect vector and tensor components. The Einstein equation gives the following equation for the evolution of $h$ :

$$
\begin{aligned}
\frac{1}{2} \ddot{h}+ & \frac{\dot{a}}{a} \dot{h}=4 \pi G\left[4 \dot{\bar{\phi}}_{q} \delta \dot{\phi}_{q}-2 \delta V\left(\phi_{q}, \rho_{x}\right)\right. \\
& \left.+\delta \rho_{x}+\delta \rho_{J}+3 \delta P_{J}\right]
\end{aligned}
$$

where $\delta \rho_{x}$ is the fluctuation of $X$ particle density, and $\delta \rho_{J}$ and $\delta P_{J}$ are, respectively, the collective density and pressure fluctuation of other fields. From the Lagrangian (1), the dynamic equation of $\phi_{q}$ is

$$
\partial_{\mu}\left(\sqrt{-g} g^{\mu \nu} \partial_{\nu} \phi_{q}\right)+\sqrt{-g} V^{\prime}\left(\phi_{q}, \phi_{x}, J\right)=0 .
$$

This equation and the energy momentum conservation determine the evolution of $\delta \phi_{q}(x, t)$,

$$
\begin{aligned}
\dot{\bar{\phi}}_{q}\left[\delta \ddot{\phi}_{q}+\partial_{i} \partial^{i}\left(\delta \phi_{q}\right)+V_{q}^{\prime \prime}\left(\bar{\phi}_{q}\right) \delta \phi_{q}+2 g\left(\frac{2 \bar{\rho}_{x}}{m_{x}^{2}}\right) \delta \phi_{q}+3 \frac{\dot{a}}{a} \delta \dot{\phi}_{q}\right]+\frac{2 g \bar{\phi}_{q}}{m_{x}^{2}}\left[2 \frac{\dot{\rho}_{x}}{\bar{\rho}_{x}} \delta \phi_{q}+\bar{\phi}_{q} \frac{\delta \dot{\rho}_{x}}{\bar{\rho}_{x}}\right] \\
\quad-\frac{\dot{a}}{a}\left\{h\left(\frac{1}{2} \dot{\bar{\phi}}_{q}^{2}-V\left(\bar{\phi}_{q}\right)\right)-6\left[V_{q}^{\prime} \delta \phi_{q}+\frac{2 g \bar{\phi}_{q} \bar{\rho}_{x}}{m_{x}^{2}}\left(2 \delta \phi_{q}+\bar{\phi}_{q} \frac{\delta \rho_{x}}{\bar{\rho}_{x}}\right)\right]\right\}-\frac{\dot{h}}{2} \dot{\bar{\phi}}_{q}^{2} \\
=\Gamma_{q}\left(\delta \rho_{x}-\frac{\delta \dot{\phi}_{q}}{\dot{\bar{\phi}}_{q}} \bar{\rho}_{x}\right) .
\end{aligned}
$$

Like the homogeneous case, we assume that SDM behaves like a pressureless fluid,

$$
T_{x}^{00}=\bar{\rho}_{x}+\delta \rho_{x}, \quad T_{x}^{0 i}=\bar{\rho}_{x} \delta u_{x}^{i}, \quad T_{x}^{i j}=\mathcal{O}\left(\delta^{2}\right) \approx 0,
$$

where $\delta u_{x}^{i}$ is the velocity of SDM fluctuations with respect to homogeneous Hubble flow. Interaction terms are explicitly included in the energy-momentum conservation equation,

$$
\begin{aligned}
\partial_{0}\left(\frac{\delta \rho_{x}}{\bar{\rho}_{x}}\right)+\partial_{i}\left(\delta u_{x}^{i}\right)-\frac{\dot{h}}{2} \\
\quad=-\pi^{4} g^{2}\left(\frac{3 \delta \rho_{x}}{m_{x}^{3}}-\frac{2 \bar{\rho}_{q}^{\prime} \delta \rho_{q}^{\prime}}{m_{q}^{3} \bar{\rho}_{x}}-\frac{\bar{\rho}_{q}^{\prime 2} \delta \rho_{x}}{m_{q}^{3} \bar{\rho}_{x}^{2}}\right) .
\end{aligned}
$$

The effect of interactions in the right-hand side of Eq. (32) is, however, very small, first because $X$ particle mass is very large and then because only high-energy $\phi_{q}$ particles contribute to this term, and their energy decreases with expansion of the Universe much faster than the SDM. The evolution of matter fluctuations is then practically the same as the standard $\Lambda$ CDM case.

Using the conservation relation for other components of the energy momentum in the limit when $\dot{\bar{\phi}}_{q} \rightarrow 0$, we find the following relation between spatial fluctuation of $\delta \phi_{q}$ and $\delta u_{x}^{i}$ :

$$
-V^{\prime}\left(\bar{\phi}_{q}, \bar{\rho}_{x}\right) \partial^{i}\left(\delta \phi_{q}\right)=\Gamma_{q} \bar{\rho}_{x} \delta u_{x}^{i}
$$

Equation (30) has a meaningful limit when $\dot{\bar{\phi}}_{q} \rightarrow 0$ only if 

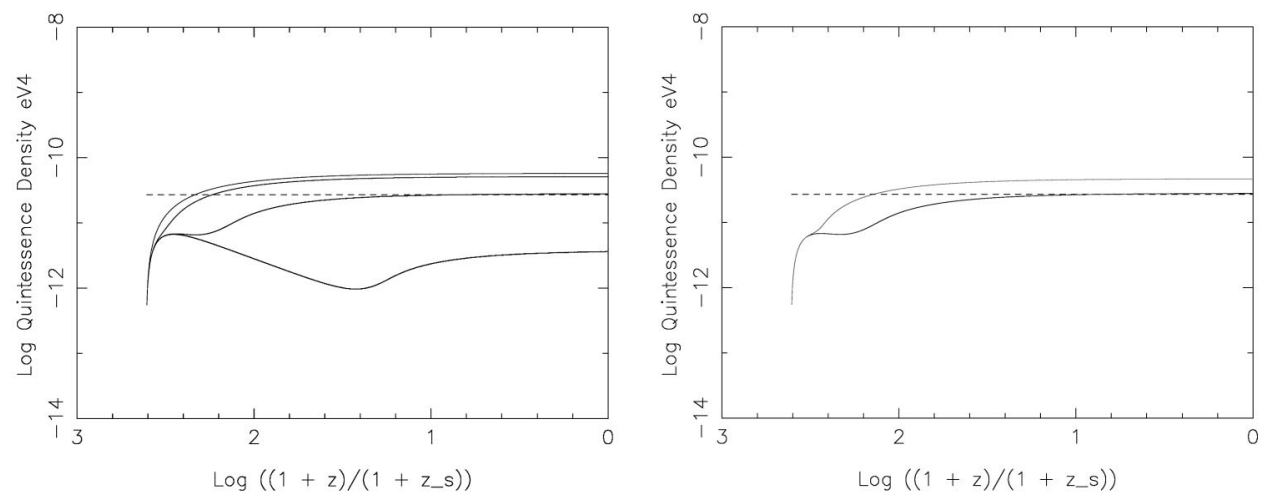

FIG. 4. Variation of quintessence energy density with mass and self-interaction. From bottom to top (dark to light gray), left: $m_{q}$ $=10^{-8} \mathrm{eV}, m_{q}=10^{-6} \mathrm{eV}, m_{q}=10^{-5} \mathrm{eV}$, and $m_{q}=10^{-3} \mathrm{eV}, \lambda=10^{-20}$; right: $\lambda=10^{-25}, \lambda=10^{-20}, \lambda=10^{-15}$, and $\lambda=10^{-10}, m_{q}$ $=10^{-6} \mathrm{eV}$. The difference between quintessence density for the first three values of $\lambda$ is smaller than the resolution of the plot. The dashed line is the observed energy density of the dark energy.

$\delta \dot{\phi}_{q} \rightarrow 0$. On the other hand, Eq. (33) shows that the divergence of quintessence field fluctuations $\partial^{i} \delta \phi_{q}$ follows the velocity dispersion of the dark matter with the opposite direction. Their amplitude, however, is largely reduced due to the very small decay width $\Gamma_{q}$. In addition, with the expansion of the Universe, $V^{\prime}\left(\bar{\phi}_{q}, \bar{\rho}_{x}\right)$ varies only very slightlyjust the interaction between SDM and $\phi_{q}$ will change. In contrast, $\bar{\rho}_{x}$ decreases by a factor of $a^{-3}(t)$, and even a gradual increase of the dark matter clumping and therefore the velocity dispersion $\delta u_{x}^{i}$ [29] cannot eliminate the effect of decreasing density. We conclude that the spatial variation of $\phi_{q}$ is very small from the beginning and is practically unobservable.

\section{CLOSING REMARKS}

Since the original works on the production of superheavy particles after inflation [12], a number of investigations [23] have demonstrated that even with a reheating temperature as low as a few $\mathrm{MeV}$, the production of superheavy particles is possible. We do not discuss here the particle physics candidates for $\phi_{q}$, but for the sake of completeness we just mention that axionlike particles are needed or at least can exist in a large number of particle physics models (see [31] for some examples). The fact that $\phi_{q}$ does not need to have very special potential is one of the advantages of this model with respect to others, and opens the way to a larger number of particle physics models as candidates for the quintessence field.

One of the arguments that is usually raised in the literature against a decaying dark matter is the observational constraints on the high-energy gamma-ray and neutrino background. In [28], it has been shown that if $m_{x} \geqslant 10^{22} \mathrm{eV}$ and its lifetime $\tau \gtrsim 5 \tau_{0}$, and if simulations correctly take into account the energy dissipation of the high-energy remnants, the present observational limits are larger than the expected flux from a decaying UHDM. Consequently, the model is consistent with the available data.

The same fact is applied to the CMB and its anisotropy. The expected CMB distortion is of order $10^{-8}$, much smaller than the sensitivity of present and near-future measurements. As for the expected anisotropy in the arrival direction of UHECR's, the data are still too scarce to give any conclusive answer. In the next few years, the Auger Observatory [30] will be able to test top-down models for UHECR's, which is one of the principal motivations for the quintessence model proposed here.

Although the limit on the amount of hot DM cannot constrain this model, a better understanding of its contribution to the total density and its content can help to understand the physics and the nature of SDM if it exists.

Evidently, the observation of $w_{q}$ and its cosmological evolution is crucial for any model of dark energy. Observation of small anisotropy in the DE density and its correlation with matter anisotropy also can be used as a signature of the relation/interaction between DM and DE.

For the range of expected masses for $\phi_{q}$ in this model, the high-energy component of the quintessence field is still relativistic. As we have discussed in Sec. II, the production of this component from annihilation has been stopped very early in the history of the Universe, and the contribution from the decay of $X$ is much smaller than the limits on the amount of hot dark matter (as has been shown in [28] for hot SM remnants). The small coupling of $\phi_{q}$ with SM particles also suppresses the probability of its direct detection. However, the detection of an axionlike particle, e.g., the QCD axion, can be a positive sign for the possibility of the existence of $\phi_{q}$-like particles in Nature.

\section{APPENDIX}

Here we calculate the right-hand side of the Boltzmann equation at lowest order of the $g$ coupling constant for annihilation and reproduction of $X$ particles.

The Boltzmann equation for $X$ particles is the following:

$$
\begin{aligned}
& p^{\mu} \partial_{\mu} f^{(X)}(x, p)-\Gamma_{\nu \rho}^{\mu} p^{\nu} p^{\rho} \frac{\partial f^{(X)}}{\partial p^{\mu}} \\
& \equiv L[f]=-[\mathcal{A}(x, p)+\mathcal{B}(x, p)] f^{(X)}(x, p)+\mathcal{C}(x, p),
\end{aligned}
$$


$\mathcal{A}(x, p)=\Gamma p^{\mu} u_{\mu}$,

$$
\begin{aligned}
\mathcal{B}(x, p)= & \frac{1}{(2 \pi)^{3} g_{x}} \sum_{i} \int d \bar{p}_{x} f^{(i)}\left(x, p_{x}\right) A \sigma_{x i}, \\
& i=X, \phi_{q}, \\
\mathcal{C}(x, p)= & \frac{1}{(2 \pi)^{6} g_{x}} \int d \bar{p}_{q} d \bar{p}_{q} f^{(q)}\left(x, p_{q}\right) \\
& \times f^{(q)}\left(x, p_{q}^{\prime}\right) A \frac{d \sigma_{\phi_{q}+\phi_{q} \rightarrow X+X}}{d \bar{p}}, \\
A= & \sqrt{\left(p_{1} p_{2}\right)^{2}-m_{1}^{2} m_{2}^{2}} .
\end{aligned}
$$

The function $f^{(X)}(x, p)$ is the distribution of $X$ particles. The terms $\mathcal{A}, \mathcal{B}$, and $\mathcal{C}$ are, respectively, the decay, the annihilation (self or in interaction with other species), and production rates. We assume that the interaction of $X$ with other fields except $\phi_{q}$ is negligible. According to Lagrangian (1) with $n=2$ and $m=2$, the lowest Feynman diagrams contributing to annihilation and production are

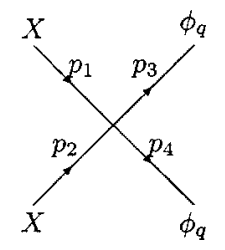

Annihilation

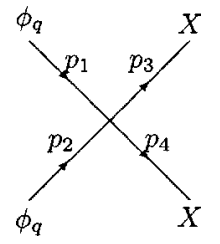

\section{Production}

The $S$ matrix for these diagrams is very simple,

$$
S=\frac{-i(2 \pi)^{4} g \delta^{(4)}\left(\sum_{i} p_{i}\right)}{\prod x_{i} 2 p_{i}^{0}}
$$

and the differential cross section

$$
d \sigma=\frac{(2 \pi)^{10} g^{2} \delta^{(4)}\left(\sum_{i} p_{i}\right)}{16 \sqrt{\left(p_{1} p_{2}\right)^{2}-m_{1}^{2} m_{2}^{2}}} d \bar{p}_{3} d \bar{p}_{4}, \quad d \bar{p}_{i} \equiv \frac{d^{3} p_{i}}{(2 \pi)^{3} g_{i} p_{i}^{0}},
$$

where $g_{i}$ is the number of internal degrees of freedom. Here we assume that $g_{x}=g_{q}=1$. Using the relation

$$
\begin{aligned}
& {\left[\int p^{\mu} p^{\mu^{1}} \cdots p^{\mu^{n}} f(x, p) d \bar{p}\right]_{; \mu}} \\
& =\int p^{\mu^{1}} \cdots p^{\mu^{n}} L[f](x, p) d \bar{p}
\end{aligned}
$$

and the definition of the energy-momentum tensor $T^{\mu \nu}$ and the number density of particles $n^{\mu}$, one obtains

$$
\begin{aligned}
T_{; \nu}^{\mu \nu}= & -\Gamma T^{\mu \nu} u_{\nu}-\pi^{4} g^{2}\left(n_{x}^{\mu} \sum_{i} \int d \bar{p}_{2} f^{(i)}\left(x, p_{2}\right)\right. \\
& -\int d \bar{p}_{1} d \bar{p}_{2} p_{1}^{\mu} f^{(q)}\left(x, p_{1}\right) f^{(q)}\left(x, p_{2}\right) \\
& \left.\times \theta\left(p_{1}^{0}+p_{2}^{0}-2 m_{x}\right)\right) .
\end{aligned}
$$

Both $f^{(X)}$ and $f^{(q)}$ have a large peak around the energies close to the mass of $X$. Therefore,

$$
\int d \bar{p} f^{(i)}(x, p) \approx \frac{n_{i}^{\nu} \bar{u}_{\nu}}{m_{i}}, \quad i=X, q
$$

In the case of $\phi_{q}$, the density $n_{q}$ is only the density of particles with an average energy larger than $m_{x}$. Finally from Eq. (A1), one can obtain the evolution equation of $\rho_{x}$ in a homogeneous cosmology, i.e., Eq. (10) in Sec. II.
[1] L. P. Chimento, A. S. Jakubi, D. Pavòn, and W. Zimdahl, Phys. Rev. D 67, 083513 (2003); L. P. Chimento, A. S. Jakubi, and D. Pavòn, ibid. 67, 087302 (2003).

[2] L. P. Chimento, A. S. Jakubi, and D. Pavòn, Phys. Rev. D 62, 063508 (2000); L. P. Chimento, A. S. Jakubi, and N. A. Zuccala, ibid. 63, 103508 (2001).

[3] W. Zimdahl, D. Diego Pavòn, and L. P. Chimento, Phys. Lett. B 521, 133 (2001).

[4] L. Amendola, Phys. Rev. D 62, 043511 (2000); Phys. Rev. Lett. 86, 196 (2001); D. Tocchini-Valentini and L. Amendola,
Phys. Rev. D 65, 063508 (2002); L. Amendola, ibid. 67, 043512 (2003).

[5] D. Comelli, M. Pietroni, and A. Riotto, Phys. Lett. B 571, 115 (2003).

[6] C. Rubano and P. Scudellaro, Gen. Relativ. Gravit. 34, 307 (2002); A. de la Macorra and C. Stephan-Otto, Phys. Rev. D 65, 083520 (2002).

[7] D. J. H. Chung, L. L. Everett, and A. Riotto, Phys. Lett. B 556, 61 (2003).

[8] G. R. Farrar and P. J. Peebles, astro-ph/0307316. 
[9] H. Ziaeepour, astro-ph/0301640.

[10] P. Bhattacharjee, C. T. Hill, and D. N. Schramm, Phys. Rev. Lett. 69, 567 (1992).

[11] E. W. Kolb, D. Chung, and A. Riotto, hep-ph/9810361.

[12] D. Chung, E. W. Kolb, and A. Riotto, Phys. Rev. D 59, 023501 (1999); V. Kuzmin and I. Tkachev, JETP Lett. 68, 271 (1998); D. Chung, Phys. Rev. D 67, 083514 (2003).

[13] D. J. Bird et al., Astrophys. J. 424, 491 (1994).

[14] N. Hayashida et al., Phys. Rev. Lett. 73, 3491 (1994); Astrophys. J. 522, 225 (1999).

[15] F. Halzen and D. Hooper, Rep. Prog. Phys. 65, 1025 (2002).

[16] S. Perlmutter et al., Astrophys. J. 483, 565 (1997); 517, 565 (1999).

[17] A. Riess et al., Astron. J. 116, 1009 (1998); P. M. Garnavich, Astrophys. J. 509, 74 (1998).

[18] H. Ziaeepour, astro-ph/0002400.

[19] WMAP Collaboration, C. L. Bennett et al., Astrophys. J., Suppl. 148, 1 (2003); WMAP Collaboration, M. R. Nolta et al., astro-ph/0305097.

[20] L. Krauss, Astrophys. J. Lett. 596, L1 (2003).

[21] J. A. S. Lima, J. V. Cunha, and J. S. Alcaniz, Phys. Rev. D 68, 023510 (2003).

[22] D. Lyth, Phys. Lett. B 488, 417 (2000); 476, 356 (2000).

[23] T. Asaka, M. Kawasaki, and T. Yanagida, Phys. Rev. D 60, 103518 (1999); G. F. Giudice, E. W. Kolb, and A. Riotto, ibid. 64, 023508 (2001).

[24] T. W. B. Kibble, Phys. Rep. 67, 183 (1980); W. H. Zurek,
Nature (London) 317, 505 (1985).

[25] F. Lombardo and F. Mazzitelli, Phys. Rev. D 53, 2001 (1996); S. Habib et al., Phys. Rev. Lett. 76, 4660 (1996); F. Lombardo, F. Mazzitelli, and R. Rivers, Nucl. Phys. B672, 462 (2003), and references therein.

[26] A. A. Starobinsky, in Current Topics in Field Theory, Quantum Gravity, and Strings, edited by H. J. De Vega and N. Sanchez, Lecture Notes in Physics Vol. 226 (Springer, Berlin, 1986).

[27] S. Habib, Phys. Rev. D 46, 2408 (1992); E. A. Calzetta, B. L. $\mathrm{Hu}$, and F. Mazzitelli, Phys. Rep. 352, 459 (2001), and references therein.

[28] H. Ziaeepour, Astropart. Phys. 16, 101 (2001).

[29] SDSS Collaboration, I. Zehavi et al., Astrophys. J. 571, 172 (2002).

[30] A. Letessier-Selvon, Nucl. Phys. B (Proc. Suppl.) 118, 399 (2003); L. A. Anchordoqui, hep-ph/0306078; G. Giudize et al., Phys. Rev. D 64, 023508 (2001); D. J. H. Chung et al., ibid. 64, 043503 (2001); A. H. Campos et al., Mod. Phys. Lett. A 17, 2179 (2002); A. A. Grib and Yu. V. Pavlov, Int. J. Mod. Phys. D 11, 433 (2002).

[31] E. A. Dudas, Phys. Lett. B 325, 124 (1994); E. Kiritsis, C. Kounnas, and D. Lust, Int. J. Mod. Phys. A 9, 1361 (1994); J. Bagger, E. Poppitz, and L. Randall, Nucl. Phys. B426, 3 (1994); N. Abe, T. Moroi, and M. Yamaguchi, J. High Energy Phys. 201, 010 (2002). 